\title{
Stimulation of Human Hematopoietic Colony Formation by Recombinant Gibbon Multi-Colony-stimulating Factor or Interleukin 3
}

\author{
Colin A. Sieff," Charlotte M. Niemeyer," David G. Nathan, ${ }^{*}$ Sarah C. Ekern, ${ }^{\star}$ Frederick R. Bieber, ${ }^{\ddagger}$ Yu Chung Yang," \\ Gordon Wong," and Steven C. Clark \\ Divisions of Hematology and Pediatric Oncology,* The Children's Hospital and The Dana-Farber Cancer Institute, Boston, \\ Massachusetts 02115; Department of Pathology, ${ }^{\ddagger}$ Brigham and Women's Hospital, Boston, Massachusetts 02115; Departments of \\ Pediatrics* and Pathology, ${ }^{\ddagger}$ Harvard Medical School, Boston, Massachusetts 02115; and Genetics Institute, ${ }^{\S}$ Cambridge, \\ Massachusetts 02140
}

\begin{abstract}
Recently, the gene for a novel mammalian hematopoietic growth factor homologous to murine interleukin 3 was isolated from a gibbon $\mathbf{T}$ cell line and expressed in monkey COS cells. The factor, termed multi-colony stimulating factor (multiCSF ) or interleukin 3, is stimulatory to human target cells. We investigated the range of enriched human bone marrow and fetal liver hematopoietic progenitors responsive to multi-CSF; compared the colony types observed with those obtained in the presence of recombinant granulocyte-macrophage CSF (GMCSF); and analyzed the effects on colony formation of combining multi-CSF with GM-CSF or granulocyte-CSF (G-CSF). The results show that multi-CSF acts as a multipoietin. Alone it stimulates the formation of colonies derived from granulocyte, macrophage, eosinophil, and megakaryocyte progenitors. In combination with erythropoietin it supports the development of both erythroid and mixed colonies. Furthermore, the data show that multi-CSF is a more potent stimulus of erythroid progenitors than GM-CSF. In combination with G-CSF multi-CSF substantially increases granulocyte colony number over the number obtained with each factor alone. We conclude that multi-CSF may prove to have important therapeutic potential in vivo as a stimulus for hematopoiesis.
\end{abstract}

\section{Introduction}

A complimentary DNA (cDNA) clone encoding a novel primate hematopoietic growth factor, multilineage-colony-stimulating factor (multi-CSF), ${ }^{1}$ or interleukin 3 (IL-3), was recently isolated from a gibbon $T$ cell line (MLA-144) (1). This

Address correspondence to Dr. Sieff, Room 1630, Dana-Farber Cancer Institute, 44 Binney St., Boston, MA 02115.

Received for publication 23 January 1987 and in revised form 8 April 1987.

1. Abbreviations used in this paper: BFU-E, erythroid burst-forming units; CFU-Eo, CFU-G, CFU-GM, CFU-M, and CFU-Meg, eosinophil, granulocyte, granulocyte-macrophage, macrophage, and megakaryocyte colony-forming units, respectively; CFU-Mix, mixed colonies compressing erythroblasts and/or granulocytes, macrophages, or eosinophils; G-CSF, granulocyte-colony-stimulating factor; GM-CSF, granulocyte-macrophage CSF; IL-3, interleukin 3; IMDM, Iscove's modified Dulbecco's medium; Mo-CM, Mo lymphoblast cell line conditioned medium; multi-CSF, multilineage CSF; PHA-LCM, phytohemagglutinin-lymphocyte conditioned medium.

J. Clin. Invest.

(c) The American Society for Clinical Investigation, Inc.

0021-9738/87/09/0818/06 $\$ 2.00$

Volume 80, September 1987, 818-823 gibbon cDNA clone was used to obtain the human homologue from a human genomic library. DNA sequence analysis revealed that the coding regions of the primate and human multi-CSF genes are $96 \%$ homologous and that both genes share significant homology, particularly with the promotor sequences encoding murine IL-3.

Murine IL-3 has been shown by a number of investigators to have a broad spectrum of biological activities when tested with a variety of different murine hematopoietic cells as targets (2). The murine factor has been shown to $(a)$ induce the expression of 20-alpha-hydroxysteroid dehydrogenase in the splenic lymphocytes of nude mice (3); (b) support the proliferation of mast cells (4) and multi-CSF-dependent cell lines (5); $(c)$ induce self generation of pluripotent stem cells (6); $(d)$ and in semi-solid medium support the formation of colonies derived from multipotent, erythroid, granulocytic, macrophage, eosinophil, and megakaryocyte progenitors $(7,8)$. The discovery of a primate hematopoietin that is structurally related to murine IL-3 raises the important question of a functional relationship between the two species. In preliminary experiments, the recombinant gibbon multi-CSF proved to be capable of supporting the growth of human erythroid and myeloid colonies (1). In this study, we analyze in detail the range of human hematopoietic progenitors that are responsive to the multi-CSF for purposes of comparison with the known biological activities of murine IL-3. Secondly, we report a comparison of the colony types obtained from human progenitor cultures established in the presence of the multi-CSF with those obtained from similar cultures established in the presence of either recombinant human granulocyte-macrophage CSF (GM-CSF) or recombinant human granulocyte CSF (G-CSF). Finally, we analyze the types of colonies found in cultures grown in the presence of a mixture of multi-CSF and either GM-CSF or G-CSF.

\section{Methods}

Recombinant gibbon multi-CSF. The plasmid pCSF-MLA, which was originally identified by functional expression cloning, was used to transfect monkey cos-1 cells as described previously (9). This plasmid directs the high level expression of the gibbon multi-CSF in the cos-1 cell system. Conditioned medium from these transfections typically supports the half-maximal proliferation of primary blasts from chronic myelogenous leukemia patients at a final dilution between 1:5,000 and 1:50,000. Mock conditioned medium from cos-1 cells transfected with vector (pXM) DNA was prepared for use as a negative control.

Recombinant human GM-CSF. Highly purified GM-CSF was kindly provided by the Pilot Development Laboratory of the Genetics Institute. This preparation was prepared from medium conditioned by a Chinese hamster ovary cell line engineered to produce high levels of human GM-CSF (10) and had a specific activity of $1-4 \times 10^{6} \mathrm{U} / \mathrm{mg}$ in the chronic myelogenous leukemia blast proliferation assay (1). 
Recombinant human $G$-CSF. Several different investigators have reported the molecular cloning of the human cDNA-encoding G-CSF $(11,12)$. Two synthetic oligonucleotides were prepared (21 mers), one with an ATGGCTGGACCTGCCACCCAG sequence and the other an CTCCTGGAGGCCCTGGAAGGG one, which exactly corresponded to two different sequences of the G-CSF cDNA (nucleotides 33-53 and 392-412 in Fig. 1 of Nagata et al. [11]). These oligonucleotides were used as hybridization probes to isolate several G-CSF cDNA clones from a library prepared from messenger RNA that was isolated from a human trophoblast cell line, designated TPA-30-1 (ATCC No. CRL 1583), which was found to express high levels of G-CSF. Because the TPA-30-1 cDNA library was originally prepared in the plasmid expression vector pXM (13), we tested all of the plasmids containing G-CSF sequences for the ability to direct the high level expression of G-CSF when introduced by DNA transfection into monkey cos-1 cells. The conditioned medium from cos- 1 cells transfected with one of these plasmids was active in supporting half-maximal human granulocyte colony formation at a final dilution of 1:30,000. This conditioned medium was used for the experiments reported here.

\section{Progenitor purification}

Bone marrow. Normal human bone marrow was obtained by aspiration from healthy adult volunteers. The marrow suspension was separated over Ficoll-Paque $(1.077 \mathrm{~g} / \mathrm{cm})$ (Pharmacia Fine Chemicals, Piscataway, $\mathrm{NJ}$ ) at $400 \mathrm{~g}$ for $40 \mathrm{~min}$ at $20^{\circ} \mathrm{C}$, and the interface mononuclear cells collected, washed three times, and resuspended in Iscove's modified Dulbecco's medium (IMDM) that contained 20\% fetal calf serum (FCS).

Fetal liver. Liver from 17-23-wk abortuses were obtained from consenting parents under a protocol approved by the Brigham and Women's Hospital's Committee for the Protection of Human Subjects from Research Risk. Livers were dissected and cells teased into IMDM containing 50\% collagenase type IV (Sigma Chemical Co., St. Louis, MO) using forceps and scissors as previously described (14). The liver clumps and cell suspension were incubated at $37^{\circ} \mathrm{C}$ in $5 \% \mathrm{CO}_{2}$ for 15-30 min, and mononuclear cells were collected by separating the cell suspension on two sequential Ficoll-Paque gradients. The cells were washed three times and resuspended in IMDM/20\% FCS.

Both bone marrow and fetal liver cells were incubated overnight at $37^{\circ} \mathrm{C} / 5 \% \mathrm{CO}_{2}$ and the nonadherent cells removed. The progenitors in the nonadherent cell fraction were enriched by immunoadsorption to Ig-coated plates (panning), as previously described $(14,15)$. The cells were incubated at $4^{\circ} \mathrm{C}$ for $30 \mathrm{~min}$ with optimal concentrations of a panel of eight monoclonal antibodies directed against the following maturation antigens: myeloid: Mol (16), My8 (17) (both bone marrow only), TG1 (18), and Leu M1 (Becton-Dickinson \& Co., Mountain View, CA); erythroid: glycophorin A (19); and lymphoid: Leu 1, 5, and 12 , Leu 10 (fetal liver only), and common acute lymphoblastic leukemia antigen (fetal liver only) (Becton-Dickinson \& Co). The cells were washed twice in IMDM containing $2 \%$ FCS and once in phosphatebuffered saline (PBS)/5\% FCS, resuspended in PBS/5\% FCS, and incubated at $4^{\circ} \mathrm{C}$ for $1 \mathrm{~h}$ on Petri dishes (Fisher Scientific Co., Pittsburgh, PA) that had been previously coated with rabbit anti-mouse $\mathrm{Ig}(20)$. Antibody-negative cells were removed and washed twice by gently swirling, tilting, and decanting. A second incubation of bone marrow cells on another antibody-coated plate was carried out to ensure removal of all antibody-labeled cells.

\section{Culture procedures}

Erythroid, myeloid, and mixed colonies. The bone marrow and fetal liver cells were cultured in a mixture containing $30 \% \mathrm{FCS}, 1 \%$ bovine serum albumin (BSA; Sigma Chemical Co.), $10^{-4}$ M 2-mercaptoethanol (Sigma Chemical Co.), penicillin-streptomycin, and $0.9 \%$ methylcellulose. Varying concentrations of the conditioned media to be tested or Mo lymphoblast cell line conditioned medium (Mo-CM) as a positive control (provided by Drs. D. Golde and J. Gasson, University of California at Los Angeles, School of Medicine, Los Angeles, CA) were added on day 0 , and on day 3 human urinary erythropoietin
(Terry Fox Laboratories, Vancouver, British Columbia, Canada) at 2 $\mathrm{U} / \mathrm{ml}$ was added to the cultures. This source of erythropoietin was identical in its effects to those of recombinant erythropoietin. The final cell concentration was $5 \times 10^{3} / \mathrm{ml}$ for bone marrow and $1 \times 10^{3} / \mathrm{ml}$ for fetal liver. Either 1- or $0.5-\mathrm{ml}$ duplicates were plated in $35-\mathrm{mm}$ petri dishes or in flat-bottomed 24-well tissue culture plates (Flow Laboratories, Inc., McLean, VA), respectively, and incubated at $37^{\circ} \mathrm{C}$ in a high humidity $4 \% \mathrm{CO}_{2} / 95 \%$ air incubator. Erythroid burst-forming units (BFU-E), granulocyte, macrophage, granulocyte-macrophage, and eosinophil colony-forming units (CFU-G, CFU-M, CFU-GM and CFU-Eo), and "mixed" colonies comprising erythroblasts and/or granulocytes, macrophages, or eosinophils (CFU-Mix) were counted on day 14 .

\section{Megakaryocyte CFU (CFU-Meg)}

Human plasma from normal donors that had been prescreened for the ability to support the proliferation of CFU-Meg was used. It was obtained from heparinized blood $(10 \mathrm{U} / \mathrm{ml})$ that had been immediately separated by centrifuging at $1,400 \mathrm{rpm}$ for $15 \mathrm{~min}$ at $20^{\circ} \mathrm{C}$. The plasma was then aspirated and centrifuged at $3,000 \mathrm{rpm}$ for $15 \mathrm{~min}$ at $20^{\circ} \mathrm{C}$. The platelet-free plasma was filtered and stored at $-20^{\circ} \mathrm{C}$ until required.

Phytohemagglutinin-lymphocyte conditioned medium (PHALCM). Phytohemagglutinin-P (Difco Laboratories, Inc., Detroit, MI) was added at $0.15 \%$ vol/vol to Ficoll-Paque-separated blood mononuclear cells cultured at $2.5 \times 10^{5} / \mathrm{ml}$ in IMDM $/ 10 \%$ FCS. The conditioned medium was collected at $72 \mathrm{~h}$ and stored at $-20^{\circ} \mathrm{C}$ until required.

Enriched bone marrow cells were cultured in a mixture containing $30 \%$ human plasma, $5 \times 10^{-5} \mathrm{M}$ mercaptoethanol, penicillin-streptomycin, $1.3 \mathrm{U}$ erythropoietin, and $0.9 \%$ methylcellulose. The multiCSF COS cell supernatant or PHA-LCM was added to the cultures at the concentrations indicated. CFU-Meg were identified and counted on day 14 .

\section{Identification of colonies}

Selected colonies from both the erythroid-myeloid and the megakaryocyte culture systems were plucked and resuspended in $200 \mu \mathrm{l}$ IMDM $/ 2 \%$ FCS. The dispersed cells were cytocentrifuged and then stained with Wright Giemsa for identification of erythroblasts, granulocytes, monocytes, and megakaryocytes. Fast green or luxol fast blue was used to confirm the identity of eosinophil colonies.

\section{Results}

Multi-CSF induces colony formation by erythroid, myeloid, mixed and megakaryocytic progenitors. Figure 1 illustrates the results from one of two similar experiments that show the responsiveness of enriched human fetal liver progenitor cells to varying concentrations of the recombinant gibbon multi-CSF. Colonies derived from BFU-E, CFU-GM, and CFU-Mix were easily detected in cultures established in the presence of the multi-CSF but not in the mock control (data not shown) providing further confirmation that the gibbon protein is indeed a multipoietin. The figure also demonstrates that about $20 \%$ of the cultured cells were capable of forming colonies. Interestingly, the concentration of factor required for optimal erythroid burst formation was more than ten fold lower than that required for optimal granulocyte/macrophage colony formation. The responsiveness of the different myeloid progenitors found in the fetal liver cultures is illustrated in Fig. 2. Colonies containing granulocytes, monocytes and eosinophils were readily identifiable by morphology and confirmed by cytochemical staining of representative individually plucked colonies. To test the effects of the multi-CSF on CFU-Meg formation, we used a slightly different culture system that had been 


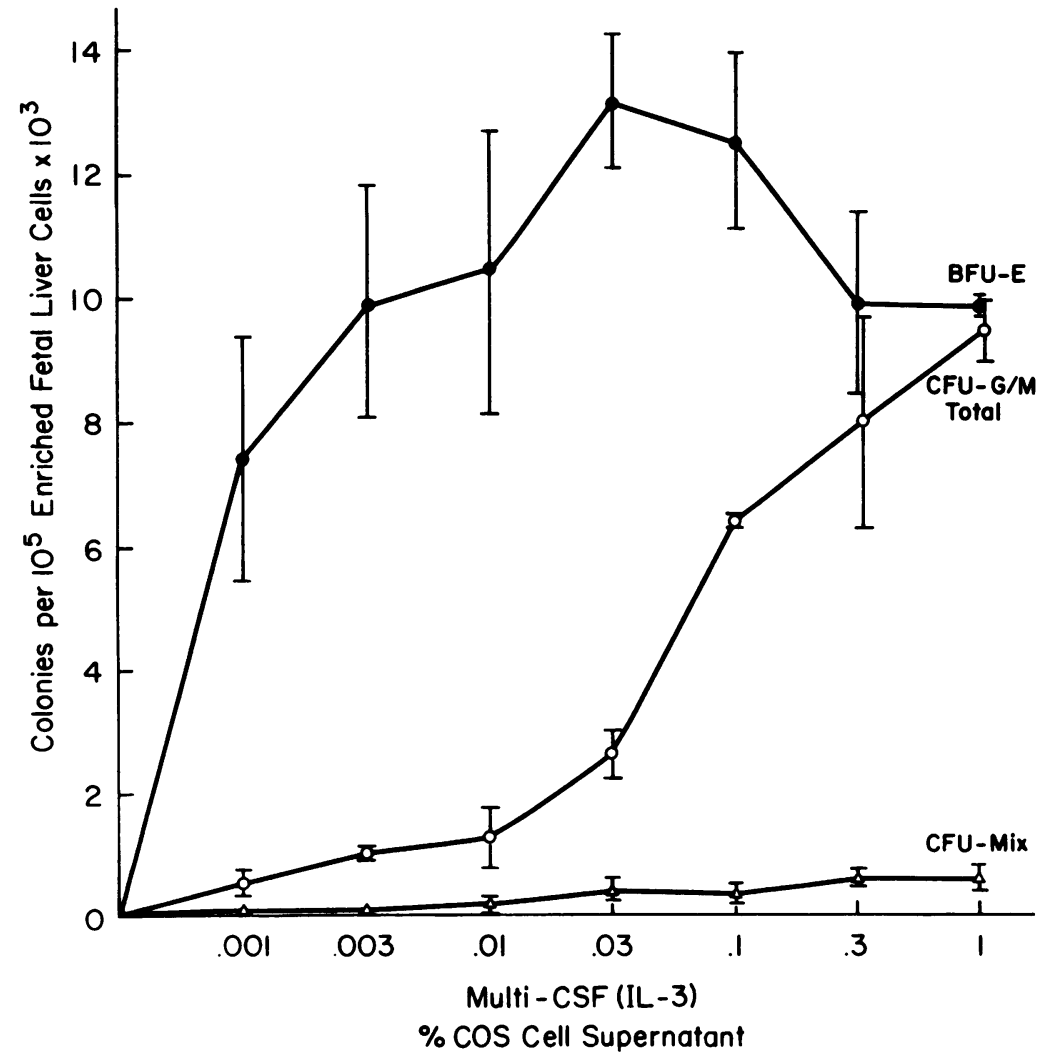

Figure 1. Stimulation of human fetal liver hematopoietic colony formation by recombinant gibbon multi-CSF. Results from one of two representative experiments in which enriched human fetal liver progenitor cells $(1,000 / \mathrm{ml})$ were plated in the presence of the indicated concentration of conditioned medium from cos-1 cells transfected with pcsf-MLA as described in Methods. BFU-E, total CFU-GM (comprising colonies containing granulocytes, macrophages, or eosinophils), and CFU-Mix (erythroblasts and/or granulocytes, macrophages, or eosinophils) were counted on day 14. Error bars represent 1 SD of the mean. optimized for the development of CFU-Meg derived colonies from enriched bone marrow progenitors (see Materials and Methods). In these experiments (Table I), the gibbon multiCSF proved as effective as mitogen-stimulated PHA-LCM in supporting human CFU-Meg formation.

Multi-CSF induces more BFU-E-derived colonies than $G M-C S F$. We have previously shown that recombinant human GM-CSF is a multi-CSF (21) that is capable of supporting colony formation by a spectrum of erythroid, myeloid, and mixed progenitors similar to that shown here to be re-

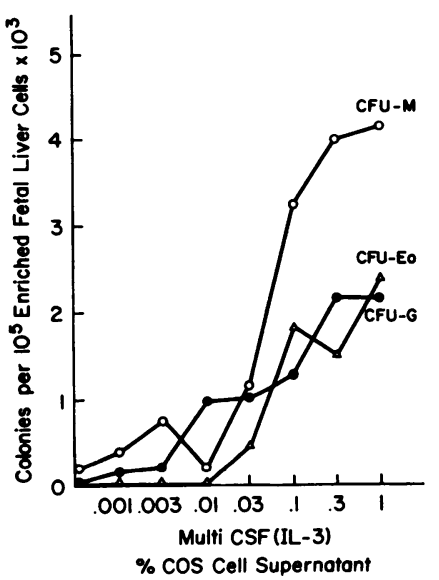

Figure 2. Responsiveness of different myeloid progenitors in fetal liver to recombinant gibbon multi-CSF. Enriched human fetal liver progenitor cultures were established as in the legend to Fig. 1 and the numbers of CFU-G, CFU-M, and CFU-Eo colonies counted on day 14. Representative colonies of each morphological subtype were individually plucked and resuspended in $200 \mu \mathrm{l}$ of IMDM $2 \%$ FCS. The dispersed cells were cytocentrifuged and stained with Wright Giemsa, fast green, or Luxol fast blue to identify granulocytes, monocytes, and eosinophils. sponsive to multi-CSF. In addition, GM-CSF is capable of supporting the proliferation of at least some megakaryocytic progenitors (C. M. Niemeyer, C. A. Sieff, unpublished observations). However, in preliminary experiments, we routinely observed greater numbers of erythroid bursts in cultures of bone marrow progenitors grown in the presence of the multiCSF than those grown in the presence of GM-CSF or with the plateau level achieved with our laboratory standard Mo-CM (Fig. 3). In this series of four experiments, the multi-CSF at optimal concentrations was found to support the proliferation of approximately $25 \%$ more BFU-E-derived colonies than Mo-CM while the plateau of erythroid colonies found in the GM-CSF-stimulated cultures was $25 \%$ less than that found in the Mo-CM controls. Comparison of these data using either a $t$-test or the Wilcoxon Rank Sum Test revealed that multi-CSF supports the formation of significantly more erythroid bursts

Table I. Multi-CSF (IL-3) Induces the Formation of CFU-Meg

\begin{tabular}{lccc} 
& & \multicolumn{2}{c}{ CFU-Meg/10 } \\
\cline { 3 - 4 } Factor & $\%$ & 1 & 2 \\
\hline Multi-CSF (IL-3) & 1 & $40^{\ddagger}$ & $10^{\ddagger}$ \\
PHA-LCM & 10 & $20^{\ddagger}$ & $15^{\ddagger}$ \\
Nil & - & 0 & 0
\end{tabular}

Effect of $1 \%$ multi-CSF (IL-3) and 10\% PHA-LCM on the formation of CFU-Meg. The data from two experiments are shown.

* Enriched bone marrow cells.

${ }^{\ddagger}$ Mean of duplicates. 


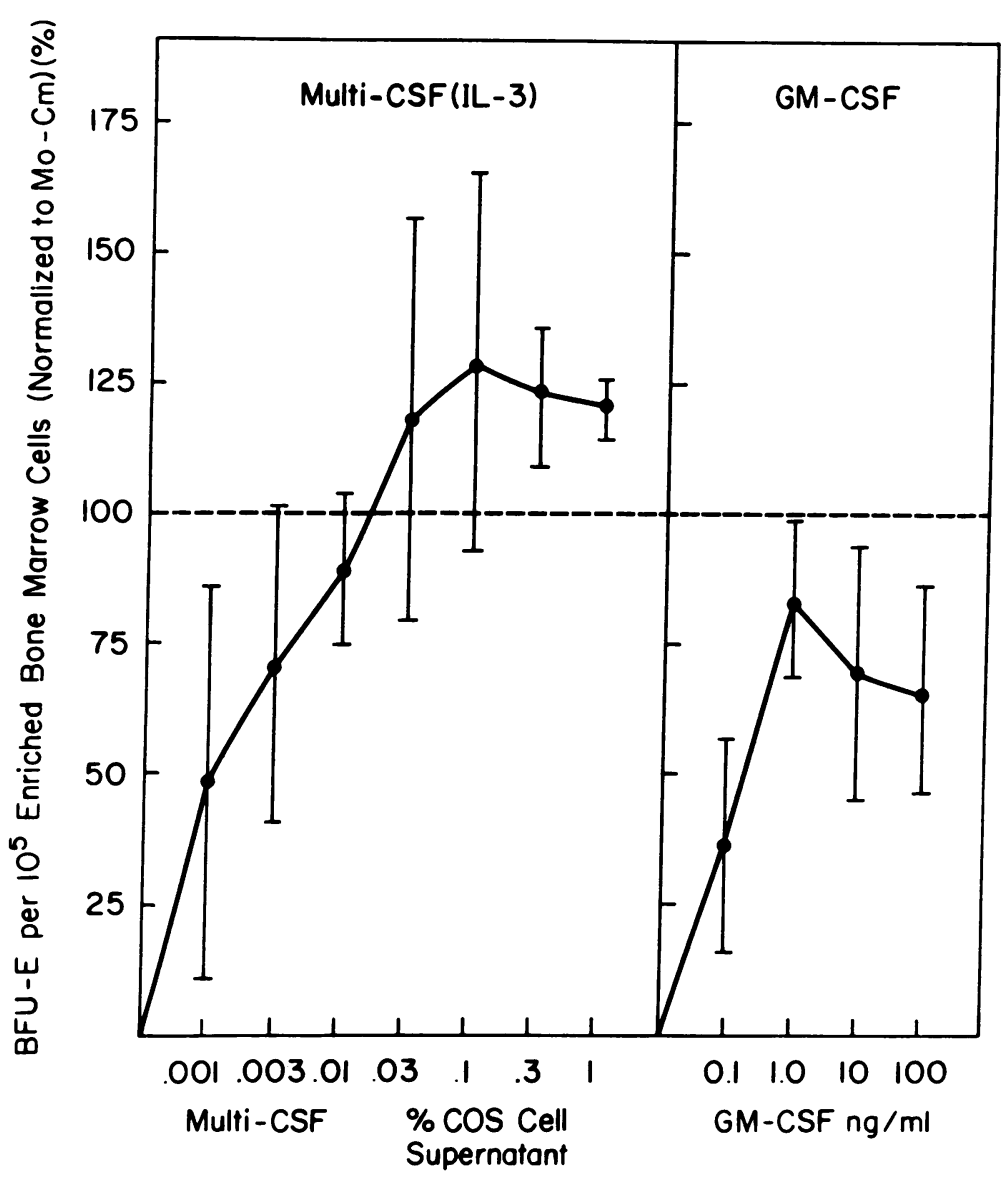

Figure 3. Comparison of the effects of gibbon multi-CSF and human GM-CSF on bone marrow BFU-E colony formation. The results from four separate experiments were normalized to the colony number observed at optimal concentrations of Mo-CM (5\% vol/vol). The same preparation of Mo-CM was used in all four experiments. The optimal value obtained with the Mo-CM was arbitrarily assigned the value $100 \%$ but the actual BFU-E number at the plateau in the four experiments varied between 200 and 3,200 per $10^{5}$ cells plated. The colony numbers obtained at plateau concentrations of multi$\operatorname{CSF}(0.03,0.1,0.3$, and $1 \%)$ were compared with those obtained with plateau concentrations of $\operatorname{GM}-\operatorname{CSF}(1,10$, and $100 \mathrm{ng} / \mathrm{ml}$ ) by the $t$ test and Wilcoxon rank sum test. At all of these concentrations, multi-CSF stimulated significantly more BFU-E colony formation than either Mo-CM $(P=0.02)$ or GM-CSF $(P<0.0001)$. Error bars represent $1 \mathrm{SD}$ of the means of the normalized results. than does GM-CSF $(P<0.0001)$. It should be noted, however, that the multi-CSF used in these experiments was a crude preparation of transfected-cos-1 cell conditioned medium while the GM-CSF was purified to homogeneity. It is possible, although unlikely, that the crude cos cell conditioned medium contained an additional activity that synergized with the multi-CSF in supporting the proliferation of a subset of BFU-E that was not responsive to GM-CSF alone.

Interactions of multi-CSF with either GM-CSF or G-CSF in supporting colony formation. Each of the different CSFs was originally characterized by the spectrum of colony types found in bone marrow cultures established in the presence of the particular factor. The observation that the different CSFs support the formation of different but overlapping sets of colony types led us to analyze colony formation in the presence of combinations of multi-CSF and either GM-CSF or G-CSF. Figure 4 shows the results obtained using the combination of multi-CSF and GM-CSF to support colony formation by enriched bone marrow progenitor cells. In this experiment the combination of factors did not result in the formation of any more BFU-E-, CFU-G-, or CFU-M-derived colonies than was observed in cultures established in the presence of multiCSF alone. The CFU-G and -M results were obtained on four occasions. Similar BFU-E results were obtained in three of four experiments. In the fourth, the combination of multi-CSF and GM-CSF induced more colonies than either factor alone. However, when the results from all four experiments were normalized for comparison, no significant stimulation was observed. The results from an experiment testing the combination of multi-CSF and G-CSF are reported in Fig. 5. In this experiment, representative of nine similar observations,

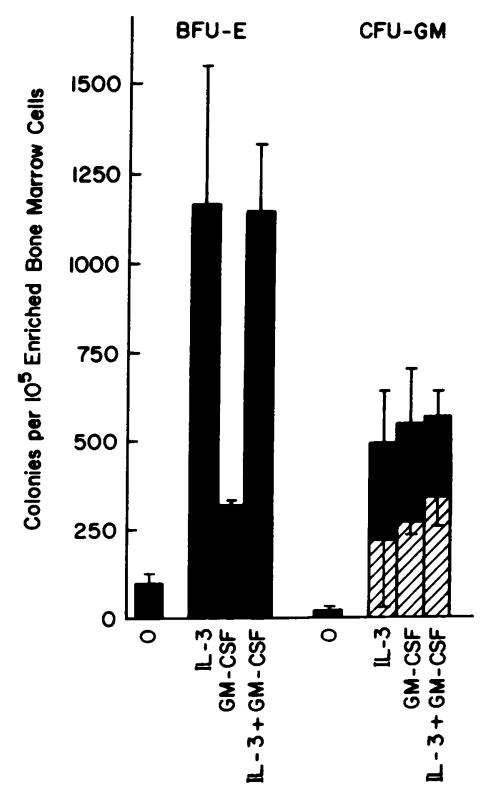

Figure 4. Stimulation of hematopoietic colony formation by multi-CSF alone and by multi-CSF in the presence of GM-CSF. One of three experiments in which similar results were obtained is shown. Enriched human bone marrow progenitors were plated in the presence of no stimulus (0), multi-CSF (IL-3, 0.1\%), or multi-CSF (IL-3, 0.1\%) and GM-CSF $(10 \mathrm{ng} / \mathrm{ml})$ as described in Methods. Multi-CSF in combination with GM-CSF induced no more BFU-E (left side of histogram, solid bars), CFU-G (right side, hatched bars), or CFU-M (right side, solid bars). The error bars represent $1 \mathrm{SD}$ of the mean of duplicate cultures. 


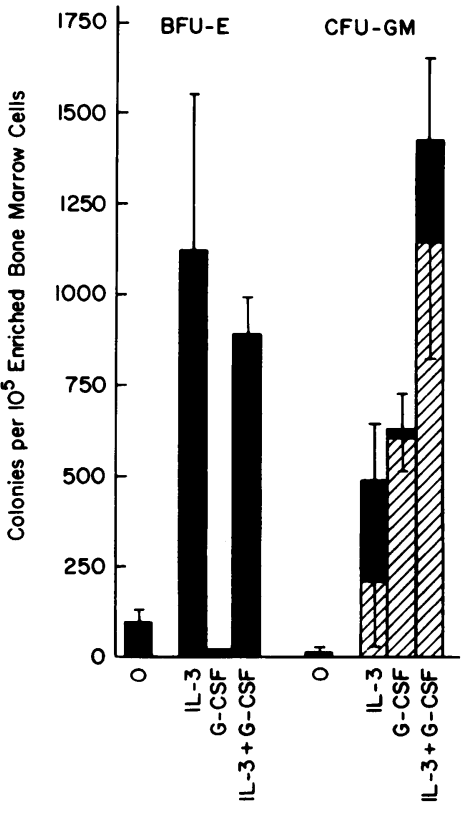

Figure 5. Stimulation of hematopoietic colony formation by multi-CSF alone and by multi-CSF in the presence of G-CSF. One of nine experiments with similar results is depicted. The cultures of enriched bone marrow progenitors were established as described in the legend to Fig. 4, except $0.03 \%$ of conditioned medium from cos- 1 cells transfected with a G-CSFexpressing plasmid was substituted for the GMCSF. The resulting colony numbers are reported as described in the legend to Fig. 4 and show that the combination of multi-CSF and G-CSF induced no increase in BFU-E or CFU-M but induced more CFU-G than either stimulus alone. The error bars represent 1 SD of the mean of duplicate cultures.

G-CSF, which does not by itself support erythroid colony formation, had no effect on BFU-E-derived colony formation supported by multi-CSF. Similarly, CFU-M-derived colony formation was not increased in cultures established in the presence of both factors relative to the number obtained with multi-CSF alone. In contrast, the combination of multi-CSF and G-CSF proved to have at least an additive effect on CFU-G-derived colonies.

\section{Discussion}

Our results confirm the observation that the novel primate hematopoietin which is structurally related to murine IL-3 does act as a multipoietin. By itself, it supported the formation of colonies derived from CFU-G, CFU-M, CFU-Eo, and CFU-Meg. In combination with erythropoietin, colonies derived from BFU-E and CFU-Mix progenitors were also obtained. In the fetal liver-derived progenitor cultures, the colony formation was observed at low cell densities $(1,000$ cells $/ \mathrm{ml})$ consistent with the model that the multi-CSF interacts directly with the target progenitor cells. Proof of this point will require further limiting dilution experiments with highly purified progenitors. However, our results suggest that the gibbon multiCSF, like the murine IL-3, acts on progenitor cells having multilineage differentiation potential as well as on later committed progenitors from several myeloid cell lineages. Further experiments will be necessary to determine the effects of the gibbon multi-CSF on earlier human pluripotent stem cells and on progenitors from the lymphoid lineages.

The multi-CSF preparations used here proved to be very effective in supporting erythroid colony formation. In fact, optimal concentrations of this factor yielded significantly more BFU-E-derived colonies than did either recombinant GM-CSF or Mo-CM. Because a mixture of multi-CSF and GM-CSF was no more effective than multi-CSF in supporting erythroid colony formation, these results distinguish two sub- populations of BFU-E: one that is multi-CSF responsive and another which is responsive to both multi-CSF and GM-CSF. Since the gibbon multi-CSF used in these experiments was not purified to homogeneity, we cannot exclude the rather unlikely possibility that the crude cos- 1 cell conditioned medium contained an activity that synergized with the multi-CSF to support colony formation by the first subpopulation of BFU-E. It will be necessary to repeat these experiments with highly purified multi-CSF to confirm that this is a unique function of multi-CSF. A similar analysis of the effects of multi-CSF on CFU-GM progenitors was not possible in these experiments because plateau levels of CFU-GM-derived colonies were often not achieved, even at the highest possible concentrations of the recombinant multi-CSF (data not shown). In our culture system, much lower concentrations of multiCSF were required to achieve optimal BFU-E colony formation than were required for CFU-GM colony formation. Whether this is due to a real difference in responsiveness of the two different types of progenitor cells or simply a reflection of suboptimal conditions for growth of CFU-GM colonies is unclear. However, the latter possibility is made less likely by the observation that the GM-CSF dose-response curves for CFUGM and BFU-E are very similar (data not shown).

In contrast to our results with combined multi-CSF and GM-CSF, we found that inclusion of G-CSF in bone marrow progenitor cell cultures along with multi-CSF resulted in a substantial increase in total colony number relative to the results obtained with the individual factors. The effect was specific for CFU-G-derived colonies: no enhancement of erythroid or macrophage colony formation was observed. The increase in the number of granulocytic colonies was found to exceed the sum of the numbers of CFU-G-derived colonies obtained with the separate factors. This result implies that there may be distinct subsets of granulocytic precursors which respond to multi-CSF, G-CSF, or the combined stimulus. Indeed, in one experiment, CFU-G were individually plucked, and colony size determined by hemocytometer counts. CFU-G contained a mean of 400 and 500 cells per multi-CSFor G-CSF-stimulated culture, respectively, while colonies derived from progenitors stimulated by both factors contained a mean of 2,800 cells. This may be analogous to the requirement of BFU-E subsets for either burst-promoting activity and erythropoietin or for erythropoietin alone (15). Further work is required to define the growth factor requirements of CFU-G progenitors more precisely.

We have shown that the recombinant gibbon multi-CSF is a potent multilineage hematopoietin active in supporting the proliferation of human progenitor cells. Because of the extensive sequence homology with the human protein, it seems likely that the human multi-CSF will display very similar biological activities. We have recently used the human gene to produce the recombinant human protein to test this expectation (Yang, Y. C., unpublished results). The availability of substantial quantities of this factor will soon permit us to begin to analyze the role of multi-CSF in regulating hematopoiesis in vivo. Murine multi-CSF has recently been shown to stimulate murine hematopoiesis in vivo $(22,23)$. In particular, it will be of great interest to compare the effects of administration of multi-CSF with those already achieved in primates by administration of GM-CSF (24). We expect that these and the other colony-stimulating factors will soon prove to be useful tools for stimulating hematopoiesis in a variety of clinical settings. 


\section{Acknowledgments}

We thank P. Temple, A. Ciarletta, and J. Witek-Giannotti for excellent technical assistance, Janet Anderson for statistical help, and Carolyn Gregory for preparation of the manuscript.

This research was supported by grants from National Institutes of Health, the Dyson Foundation, and the Deutsche Forschungsgemeinschaft.

\section{References}

1. Yang, Y. C., A. B. Ciarletta, P. A. Temple, M. Chung, S. Kovacic, J. S. Witek-Giannotti, A. C. Leary, R. Kriz, R. E. Donahue, G. G. Wong, and S. C. Clark. 1986. Human interleukin-3 (multi-CSF): identification by expression cloning of a novel hematopoietic growth factor related to murine IL-3. Cell. 47:3-10.

2. Metcalf, D. 1984. The Hemopoietic Colony-stimulating Factors. Elsevier, Amsterdam. 74-80.

3. Ihle, J. N., L. Petersack, and L. Rebar. 1981. Regulation of T cell differentiation: in vitro reduction of 20 alpha-hydroxysteroid dehydrogenase in splenic lymphocytes and mediated by a unique lymphokine. J. Immunol. 126:2184-2189.

4. Ihle, J. N., J. Keller, S. Oroszlan, L. E. Henderson, T. D. Copeland, F. Fitch, M. B. Prystowsky, E. Goldwasser, J. Schrader, E. Palaszynski, M. Dy, and B. Lebel. 1983. Biological properties of homogeneous interleukin 3. 1. Demonstration of WEHI-3 growth factor activity, mast cell growth factor activity, P-cell stimulating activity, colony stimulating activity and histamine-producing cell-stimulating activity. J. Immunol. 131:282-287.

5. Bazill, G. W., M. Haynes, J. Garland, and T. M. Dexter. 1983. Characterization and partial purification of a haemopoietic cell growth factor in WEHI-3 cell conditioned medium. Biochem. J. 210:747-759.

6. Iscove, N. N., C. A. Roitsch, N. Williams, and L. J. Guilbert. 1982. Molecules stimulating early red cell, granulocyte, macrophage, and megakaryocyte precursors in culture: similarity in size, hydrophobicity, and charge. J. Cell. Physiol. 1:65-78.

7. Clark-Lewis, I., S. B. H. Kent, and J. W. Schrader. 1984. Purification to apparent homogeneity of a factor stimulating the growth of multiple lineages of hemopoietic cells. J. Biol. Chem. 259:7488-7494.

8. Goldwasser, E., J. N. Ihle, M. B. Prystowsky, I. Rich, and G. Van Zant. 1983. The effect of interleukin-3 on hemopoietic precursor cells. In Normal and Neoplastic Hemopoiesis, UCLA Symposia on Molecular and Cellular Biology. D. W. Golde and P. A. Marks, editors. Alạn R. Liss, Inc., New York. 301-309.

9. Gluzman, Y. 1981. SV40-transformed simian cells support the replication of early SV40 mutants. Cell. 23:1339-1342.

10. Donahue, R. E., E. A. Wang, R. J. Kaufman, L. Foutch, A. C. Leary, J. S. Witek-Giannotti, M. Metzer, R. M. Hewick, D. R. Steinbrink, G. Shaw, R. Kamen, and S. C. Clark. 1986. Effects of N-linked carbohydrate on the in vivo properties of human GM-CSF. Vol. 51. Cold Spring Harbor Symposium of Quantitative Biology, Cold Spring Harbor, NY. 685-692.

11. Nagata, S., M. Tsuchiya, S. Asano, Y. Kaziro, T. Yamazaki, O. Yamamoto, Y. Hirate, N. Kubota, M. Oheda, H. Nomura, and $M$.
Ono. 1986. Molecular cloning and expression of cDNA for human granulocyte colony-stimulating factor. Nature (Lond.). 319:415-418.

12. Souza, L. M., T. C. Boone, J. Gabrilove, P. H. Lai, K. M. Zsabo, D. C. Murdock, V. R. Chazin, J. Bruszewski, H. Lu, K. K. Chen, J. Barendt, E. Platzer, M. A. S. Moore, R. Mertelsmann, and K. Welte. 1986. Recombinant human granulocyte colony-stimulating factor: effects on normal and leukemic myeloid cells. Science (Wash. DC). 232:61-65.

13. Wong, G. G., P. A. Temple, A. C. Leary, J. S. Witek-Giannotti, Y.-C. Yang, A. B. Ciarletta, M. Chung, P. Murtha, R. Kriz, R. J. Kaufman, C. R. Ferenz, B. S. Sibley, K. J. Turner, R. M. Hewick, S. C. Clark, N. Yanai, H. Yokota, M. Yamada, M. Saito, and K. Motoyoshi. 1986. Human CSF-1: molecular cloning and expression of a $4 \mathbf{~ k b}$ cDNA encoding the human urinary protein. Science (Wash. DC). 235:1504-1508.

14. Emerson, S. G., C. A. Sieff, E. A. Wang, G. G. Wong, S. C. Clark, and D. G. Nathan. 1985. Purification of fetal hemopoietic progenitors and demonstration of recombinant multipotential colonystimulating activity. J. Clin. Invest. 76:1286-1290.

15. Sieff, C. A., S. G. Emerson, A. Mufson, T. G. Gesner, and D. G. Nathan. 1986. Dependence of highly enriched human bone marrow progenitors on hemopoietic growth factors and their response to recombinant erythropoietin. J. Clin. Invest. 77:74-81.

16. Todd, R. F., III, L. M. Nadler, and S. F. Schlossman. 1981. Antigens on human monocytes identified by monoclonal antibodies. J. Immunol. 126:1435-1442.

17. Griffin, J. D., J. Ritz, L. M. Nadler, and S. F. Schlossman. 1981. Expression of myeloid differentiation antigens on normal and malignant cells. J. Clin. Invest. 68:932-941.

18. Beverley, P. C. L., D. Linch, and D. Delia. 1980. Isolation of human hematopoietic progenitor cells using monoclonal antibodies. Nature (Lond.). 287:332-333.

19. Bigbee, W. L., M. Vanderlaan, S. S. N. Fong, and R. H. Jensen. 1983. Monoclonal antibodies specific for the $M$ and $N$ forms of human glycophorin A. Mol. Immunol. 20:1353-1362.

20. Wysocki, L. J., and V. L. Sato. 1978. "Panning" for lymphocytes: a method for cell selection. Proc. Natl. Acad. Sci. USA. 75:2844-2848.

21. Sieff, C. A., S. G. Emerson, R. E. Donahue, D. G. Nathan, E. A. Wang, G. G. Wong, and S. C. Clark. 1985. Human recombinant granulocyte macrophage colony stimulating factor: a multi-lineage hematopoietin. Science (Wash. DC). 230:1171-1173:

22. Kindler, J., B. Thorens, S. Kossodo, B. Allet, J. F. Aliason, D. Thatcher, N. Farber, and P. Vassalli. 1986. Stimulation of hematopoiesis in vivo, by recombinant bacterial murine interleukin 3. Proc. Natl. Acad. Sci. USA. 83:1001-1005.

23. Metcalf, D., C. G. Begley, G. R. Johnson, N. A. Nicola, A. F. Lopez, and D. J. Williamson. 1986. Effects of purified bacterially synthesized murine multi-CSF (IL-3) on hematopoiesis in normal adult mice. Blood. 68:46-57.

24. Donahue, R. E., E. A. Wang, D. Stone, R. Kamen, G. G. Wong, P. K. Sehgal, D. G. Nathan, and S. C. Clark. 1986. Stimulation of hematopoiesis in primates by continuous infusion of recombinant human GM-CSF. Nature (Lond.). 321:872-875. 